\title{
Investigation of the tribology behaviour of the graphene nanosheets as oil additives on textured alloy cast iron surface
}

\author{
Dan Zheng ${ }^{1}$, Zhen-bing Cai ${ }^{1}$, Ming-xue Shen ${ }^{2}$, Zheng-yang $\mathrm{Li}^{1}$ and Min-hao Zhu ${ }^{1}$ \\ ${ }^{1}$ Tribology Research Institute, Key Lab of Advanced Technologies of Materials, Southwest Jiaotong \\ University, Chengdu, 610031, China \\ ${ }^{2}$ Engineering Research Center of Process Equipment and Its Remanufacture, Ministry of Education, \\ Zhejiang University of Technology, Hangzhou 310032, China \\ * Corresponding author: 86-02887600601, czb-jiaoda@126.com
}

\begin{abstract}
Tribological properties of graphene nanosheets (GNS) as lubricating oil additives on textured surfaces were investigated using a UMT-2 tribotester. The lubricating fluids keeping a constant temperature of $100^{\circ} \mathrm{C}$ were applied to a $\mathrm{GCr} 15$ steel ball and an $\mathrm{RTCr} 2$ alloy cast iron plate with various texture designs (original surface, dimple density of $22.1 \%, 19.6 \%$ and $44.2 \%$ ). The oil with GNS adding showed good tribological properties (wear reduced 50\%), especially on the textured surfaces (the reduction in wear was high at over 90\%). A combined effect between GNS additives and laser surface texturing (LST) was revealed, which is not a simple superposition of the two factors mentioned. A mechanism is proposed to explain for these results -the graphene layers sheared at the sliding contact interfaces, and form a protective film, which is closely related with the GNS structures and surface texture patterns
\end{abstract}

Keywords: graphene nanosheets, oil additives, laser surface texturing, wear reduction 


\section{Introduction}

High efficiency, long durability and safety: an eternal theme of moving mechanical systems. However, it has been well known for some time now that a large number of our non-renewable energy resources were used to overcome friction, and a large number of mechanical system failure originated from wear [1]. Consequently, research and industrial application of tribology has been paid more and more attentions [2]. Diana et al. [3] believe that one of the most effective ways to control or reduction friction is to use a lubricant in liquid and/or solid forms. Recently, the study of carbon nanomaterials used as lubricant additives were widely reported. Alazemi [4] et al. found that ultrasmooth submicrometer carbon spheres as lubricant additives manifested good tribological properties for friction and wear reduction. Carbon nanotubes have been studied as lubricant additives by $\mathrm{Yu}$ et al., and shown good tribological performance [5,6].

Graphene, a single atom thick carbon with a 2D structure, was derived from a single layered sheet of graphite by Novoselov and Geim first time [7], and has attracted great attention due to its unique properties (including lubricating potential). Zhang et al. [8] found that oleic acid-modified graphene shown good tribological performance in lubricating oil. The study by Kinoshita et al. [9] showed that graphene oxide sheets function as an excellent water lubricant additive to reduce friction and surface wear. Mao et al. [10] have studied that graphene as a lubricant on $\mathrm{Ag}$ for electrical contact applications. Simultaneously, friction and wear properties of different types of graphene nanosheets have been studied as effective solid lubricant by Peng et al. [11]. All of these studies showed that graphene has a potential and promising tribological application. However, the research on pure graphene used as lubricating oil additives has rarely been reported because graphene is neither hydrophilic nor lipophilic [12].

In addition, surface texturing technique is another commonly used method in surface engineering. And its friction reducing and anti-wear mechanisms have been widely reported as a decrease in real contact area, wear debris entrapment and lubricating oil storing [13-16]. Recently, various surface texturing techniques, such as mechanical machining [17], chemical etching [18], and laser texturing $[19,20]$ have been widely used to improve the tribological 
characteristics of rubbing interface. Among these, laser surface texturing (LST) offers some obvious advantages, such as versatility, fast adaptability, high precision and cleanness of environment $[19,21]$.

To date, most of the research on lubricant additives has been conducted on smooth surfaces. The tribological properties of lubricant additives on textured surfaces are worth studying and exploring. In this study, tribological properties of graphene nanosheets (GNS) as lubricating oil additives on textured surfaces were investigated on sliding steel-steel interfaces at a constant temperature of $100^{\circ} \mathrm{C}$ by using a UMT-2 ball-on-plate tribotester, and a combined effect of these two factors on reducing the friction and wear was revealed. The details were provided in the Supporting Information.

\section{Materials and methods}

\subsection{Material processing and sample preparation}

The graphene used in this study was purchased from Jinan Graphene New Materials Co., Ltd, and the trademark is MX-AVS. Its structural properties were measured by Atomic force microscopy (AFM, SPI3800N) and Raman spectroscopy (LabRam HR) equipped with a 532 nm laser.

The ball sample was GCr15 steel (wt \%: 1.0 C, $1.49 \mathrm{Cr}, 0.31 \mathrm{Mn}, 0.26 \mathrm{Si}, 0.009 \mathrm{P}, 0.004$ S) with a diameter of $9.525 \mathrm{~mm}$ and hardness of $766 \mathrm{HV}$. The plate sample was RTCr2 alloy cast iron (wt \%, 3.11 C, $2.21 \mathrm{Si}, 0.50 \mathrm{Mn}, 1.63 \mathrm{Cr}$ ) with hardness of $220 \mathrm{HV}$, which was cut into $25 \times 12 \times 6 \mathrm{~mm}$ sheets and subsequently polished to obtain a surface roughness of $\mathrm{Ra} \leq 0.03 \mu \mathrm{m}$. Micro-dimple patterns were then created on the RTCr2 plate surfaces through an Nd: YAG laser with the wavelength of $1064 \mathrm{~nm}$, the pulse frequency of $10 \mathrm{kHz}$, the average power of $10 \mathrm{~W}$ and the scanning speed of $5 \mathrm{~mm} / \mathrm{s}$. After laser texturing, all samples were re-polished gently to remove bulges or burrs around the rim of the dimples and obtain a roughness of $\mathrm{Ra} \leq 0.03 \mu \mathrm{m}$. Three dimple densities with the same dimple geometric parameters were designed. A smooth surface was also used to provide a baseline for comparison. These four plate samples were denoted as $0 \#, 1 \#, 2 \#$ and $3 \#$ respectively. The details of the parameters are shown in Table 1. 
Table 1 Characteristics of various laser textures

\begin{tabular}{ccccc}
\hline Samples & $0 \#$ & $1 \#$ & $2 \#$ & $3 \#$ \\
Pattern & Original & & & \\
Dimple density $(\%)$ & - & 22.1 & 300 & 200 \\
Dimple spacing $(\mathrm{X}, \mu \mathrm{m})$ & - & 400 & 150 & 150 \\
Dimple diameter $(\mu \mathrm{m})$ & - & 150 & 30 & 30 \\
Dimple depth $(\mu \mathrm{m})$ & - & 30 & & 300 \\
\hline
\end{tabular}

\subsection{Experimental procedure}

Tribological tests were performed using a UMT-2 tribometer in the ball-on-plate reciprocating wear mode with applied load of $10 \mathrm{~N}$, sliding distance of $8 \mathrm{~mm}$, speed of 5 $\mathrm{mm} / \mathrm{s}$, and the sliding direction parallel to the LST patterns. The upper ball sample and the lower plate sample were fixed in a square tank equipped with lubricating oil. The bottom of the tank was fitted with a heating device that can control the experimental temperature. Before each test, the balls and plates were cleaned with ethanol to keep the surfaces as clean as possible. In this study, $0.01 \mathrm{wt} \%$ GNS was added into PAO4 oil as lubricant. Considering the difficulty in preparing stable dispersion of graphene [22-24], 1 wt \% Span-80 $\left(\mathrm{C}_{24} \mathrm{H}_{44} \mathrm{O}_{6}\right.$, Chengdu, Changzheng Glass Co., Ltd.) was used as a dispersing agent, and the mixed oil was stirred for over $10 \mathrm{~min}$ and then subjected to $15 \mathrm{~min}$ of ultrasonic to create a uniform suspension in oil. PAO4 base oil was also used to provide a baseline for comparison. Two series of tribological tests, including those under PAO4 base oil lubrication and GNS dispersed oil lubrication conditions, were run for 6000 s at a constant temperature of $100^{\circ} \mathrm{C}$ with the same test parameters.

After the tests, the ball and plate samples were cleansed with ethanol. The morphologies and tribochemistry of worn surfaces were analysed through 2D profiler (NanoMap-D), optical microscopy (OM, OLYMPUS BX60M), scanning electron microscopy (SEM, JSM-7001F), energy dispersive X-ray spectroscopy (EDX, EDAX-7760/68M) and Raman spectroscopy 
(LabRam HR). To estimate the wear rate after the tests, we calculated the wear volume of the plate side as follow.

\section{Results and discussion}

\subsection{Characterization of GNS and texture}

Fig. 1a shows the AFM image of the GNS sample. The sample was prepared by dropping a $0.01 \mathrm{wt} \%$ GNS dispersion onto a freshly cleaved Si substrate followed by drying in air. The height of the GNS sample was measured at around $1.5 \mathrm{~nm}$. It concluded that the GNS had a few-layer structure. To obtain additional information on the GNS structure, Raman spectrum of the GNS sample was conducted and shown in Fig. 1b. It seen that the intensity of G peak is higher than that of 2D peak, and the 2D peak of the GNS sample can be fitted with six Lorenz peaks. It is likely that the GNS was three layers [25-27]. In summary, the GNS used in this study had a few-layer structure.

Fig. 2 shows the digital pictures of GNS dispersions with and without addition of $1 \mathrm{wt} \%$ Span-80 immediately after sonication (a) and 4 weeks after sonication (b). 0.05 wt $\%$ GNS solution was selected for better observation. After adding Span-80, the GNS additives were well dispersed in PAO4 oil, probably due to the edge sites and basal planes of GNS was coated with the surfactant Span-80, which made GNS partially oleophilic that guarantees the stable existence of GNS in PAO4 oil. However, the GNS dispersion without addition of Span-80 displayed only short-term stability and precipitated completely in a matter of hours to a few days. By contrast, it shown that the surfactant Span-80 effectively allows GNS to disperse well in PAO4 oil.

3D morphologies of plate samples with various surface textures are shown in Fig. 3. It can be seen that the micro-dimples are arranged regularly on the surfaces. 


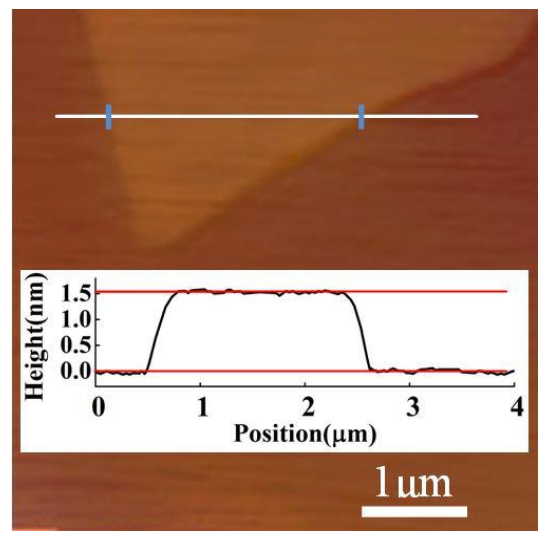

(a) AFM image

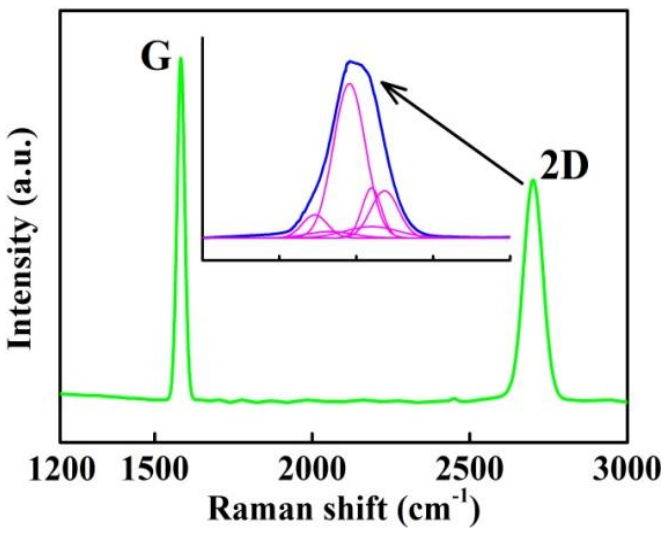

(b) Raman spectrum

Fig. 1 Characterization of the GNS.

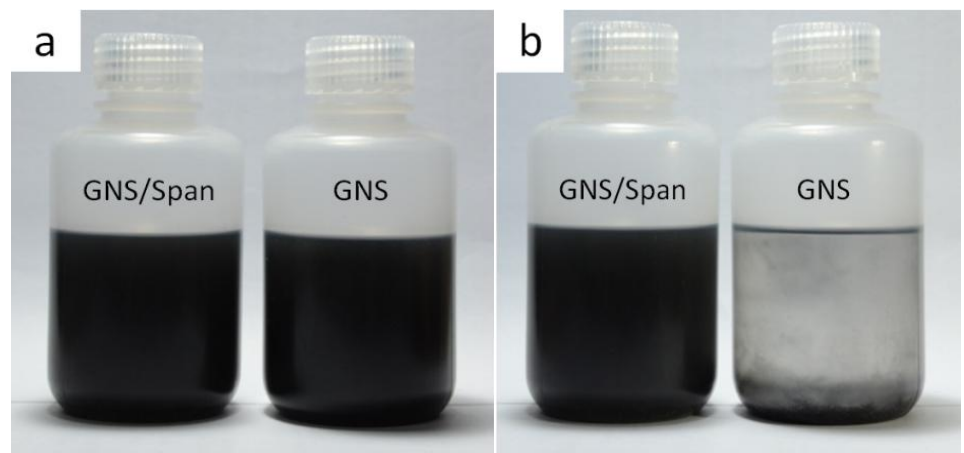

Fig. 2 Digital pictures of GNS dispersions with and without addition of 1 wt \% Span-80 immediately after sonication (a) and 4 weeks after sonication (b).
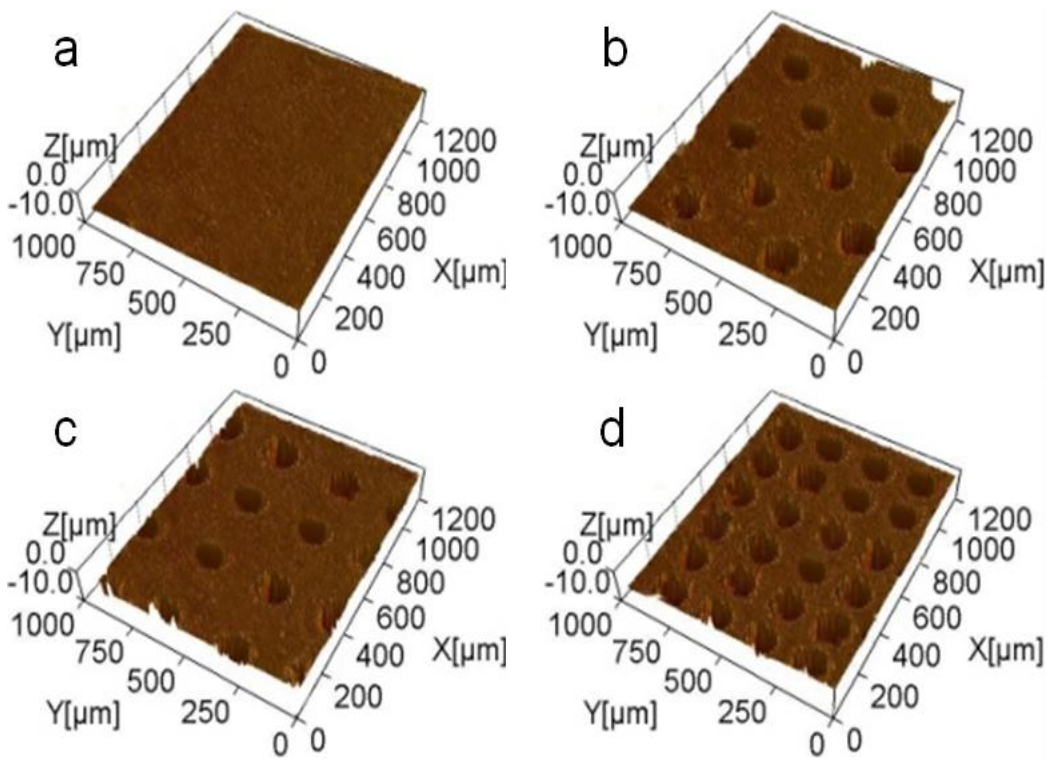

Fig. 3 3D morphologies of plate samples. (a) 0\#, (b) 1\#, (c) 2\# and (d) 3\#. 


\subsection{Friction and wear}

Fig. 4 shows the curves of the friction coefficient (COFs) of various textured surfaces under (a) PAO4 base oil and (b) GNS dispersed oil lubrication. For the condition of PAO4 base oil lubrication, the COF presented relatively high values and acute fluctuation (Fig. 4a). And surface textures resulted in higher COF, probably due to the increase in surface roughness leading to greater resistance at the sliding contact interfaces [28], implying that surface textures have effect on enhancing friction. Furthermore, the COF of $3 \#$ was significantly lower than that of $1 \#$ and $2 \#$, probably due to the lubricating oil and wear debris storing of micro-dimples [29]. As shown in Fig. 4b, friction was effectively reduced and more stable after adding GNS additives. For example, the COF of 0\# was reduced to approximately 0.29 from approximately 0.59 . This COF reduction is thought to be attributed to the combined lubrication effect of Span-80 and GNS additives. However, tests performed with Span-80 dispersed oil (PAO4 base oil +1 wt \% Span-80) lubrication showed a same COF as that of PAO4 base oil lubrication (not shown here), suggesting that the GNS is most likely the reason for the friction reduction.

In the following, the impact on wear of surface textures and GNS additives will be discussed. Considering the wear on the balls was too low to quantify, worn plate scars were mainly analysed in this study. The wear rates of plates are compared in Fig. 5; cross-sectional profiles of plate wear scars are also shown in Fig. 5. As shown, the wear rate of the original friction and lubrication condition (both surface textures and GNS additives were not used) presented a high value. However, relatively higher values of the wear rates were presented when surface texturing was used; indicating that surface texturing not only increases the friction but also increases the wear. As was expected, the wear rate was effectively reduced with the addition of GNS, for instance, the wear rate of 0\# reduced from 778 to 371 . It cannot be overlooked that the wear rates of textured plates were significantly lower than that of the original one for the tests performed in GNS dispersed oil. This finding clearly reveals the combined effect on reducing wear between GNS and surface textures, and it is not a simple superposition of the two factors mentioned. 


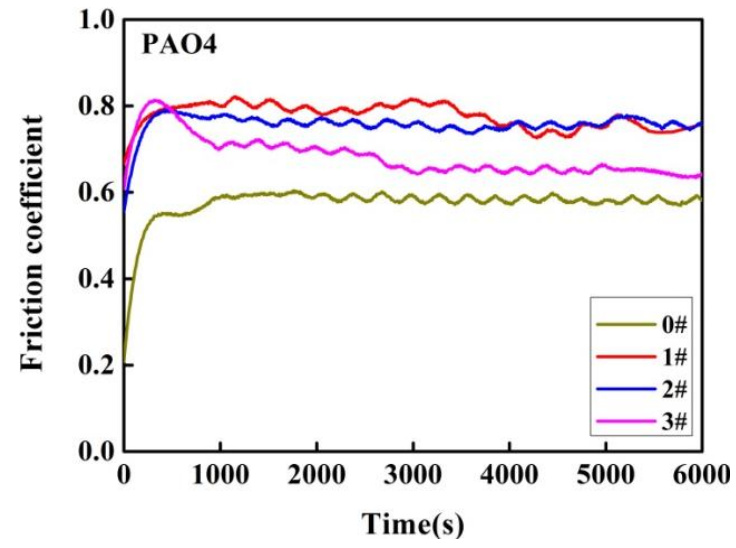

(a)

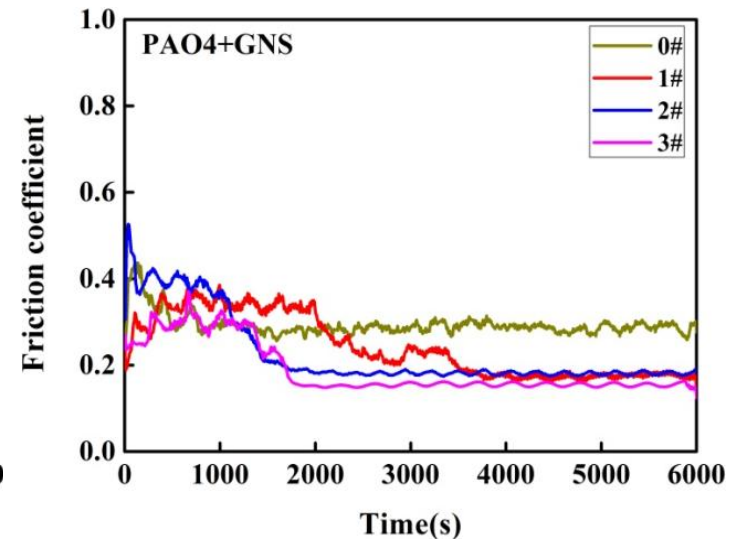

(b)

Fig. 4 Friction coefficient curves of various textured surfaces under (a) PAO4 base oil and (b) GNS dispersed oil lubrication.

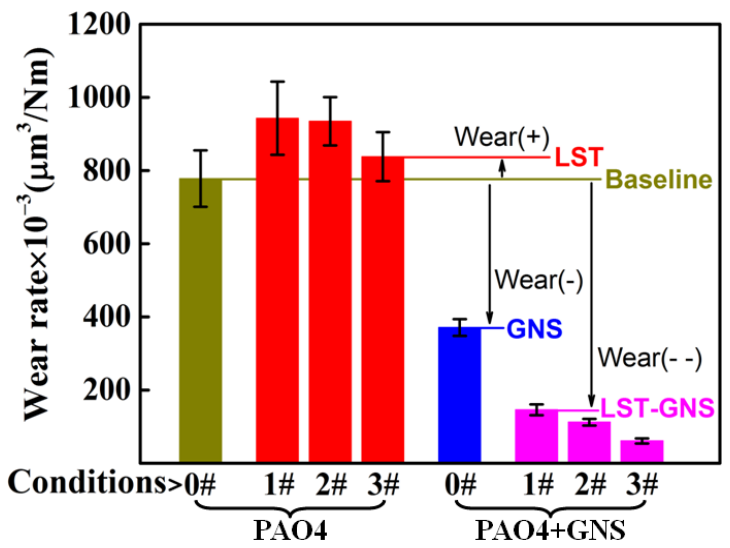

(a)

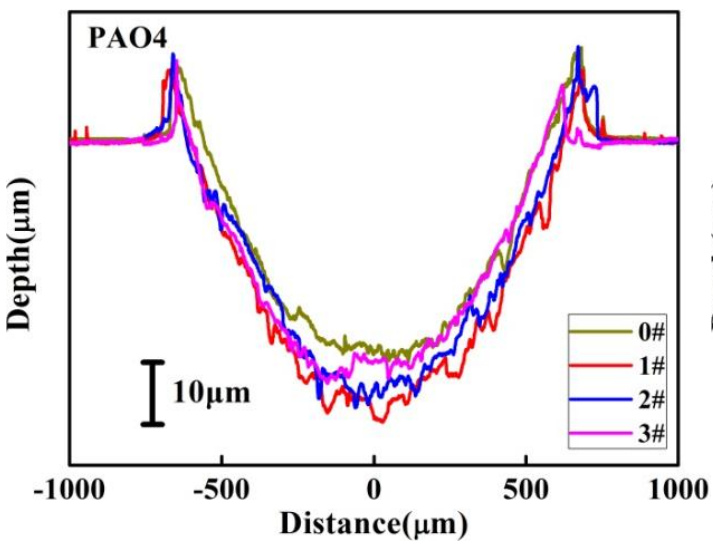

(b)

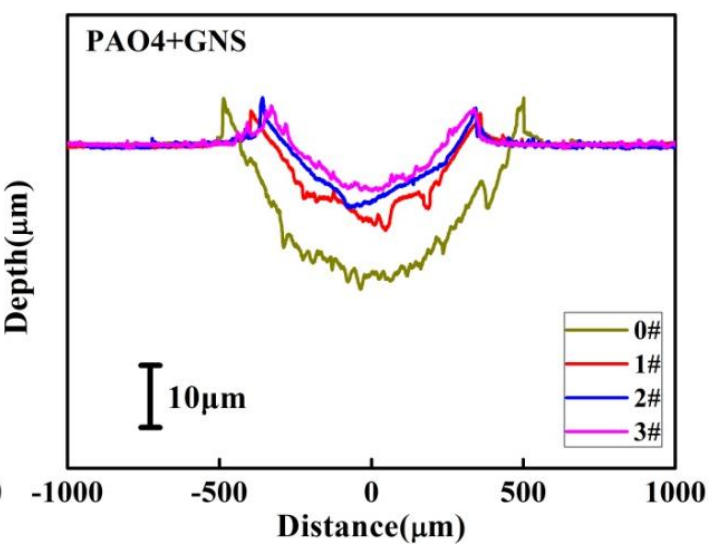

(c)

Fig. 5 Comparison of wear of plate samples with various surface textures. (a) Wear rates of the worn plates and cross-sectional profiles of plate wear scars under (b) PAO4 base oil and (c) GNS dispersed oil lubrication. 


\subsection{Morphological analysis of worn surfaces}

The optical morphologies of the worn surfaces of the balls and various textured plates tested in PAO4 oil with and without addition of GNS are presented in Fig. 6. The wear scars of balls present oval shape instead of circular shape, and the explanation have been proffered by Cai et al. [30]: the contact area of ball and plate in the sliding direction is smaller than those in the vertical direction, because the plate material is worn off in the sliding direction. For wear scars generated under GNS dispersed oil lubrication, worn single dimple can be observed on the worn ball surfaces (zones surrounded by red circles), probably due to the local high pressure on the edge of micro-dimples. You can imagine that same phenomenon may occurred in the tests performed in PAO4 base oil but cannot observed because of wearing heavily. It's not difficult to understand that micro-dimples may lead to greater contact pressure at the sliding contact interface, and it may be the reason for the increase of friction and wear resulted from surface texturing. The width of plate wear scars is also indicated in Fig. 6.

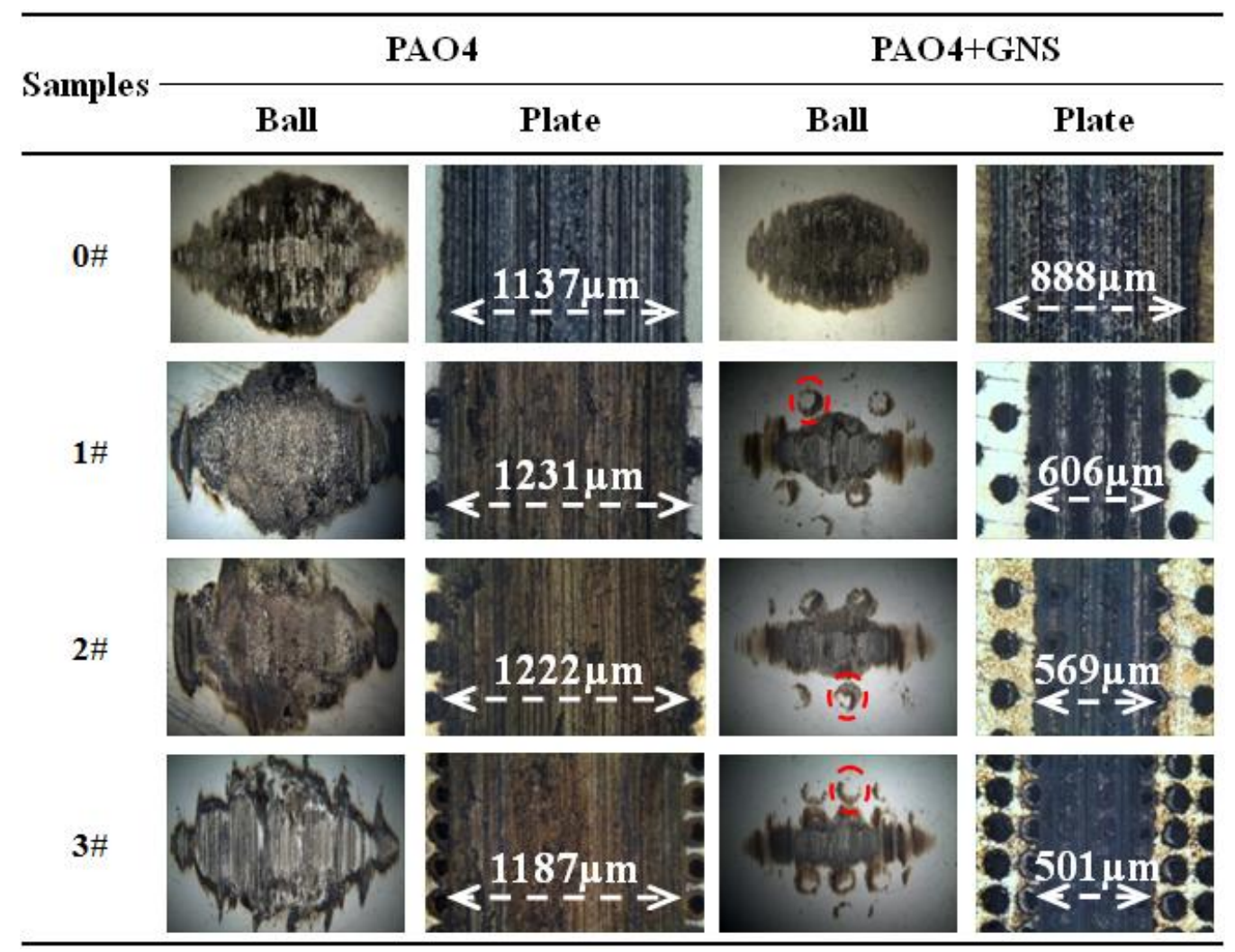

Fig. 6 Optical morphologies of worn surfaces.

The wear morphologies of various textured plates tested in PAO4 oil with and without addition of GNS were investigated by SEM. As can be seen in Fig. 7a,b, the worn scar of o\# 
under PAO4 base oil lubrication displays deep machining marks and the material spall seriously, indicating that severe scuffing occurred in this case. After adding GNS additives, the defects become relatively shallow and narrow and the worn surface becomes smoother, despite the presence of machining marks (Fig. 7c,d), showing that the GNS may offer protection to the sliding interface. Fig. 8 to Fig. 10 display the combined effect between surface texturing and GNS additives on reducing wear. When both texture and additive were used, the worn surfaces were significantly smoother and the defects were not obvious comparing with that under single effect of GNS additives. In addition, the worn surface of 2\# under GNS dispersed oil lubrication shows relatively complete residue micro-dimples and slight defects than that of $1 \#$, suggesting that $2 \#$ have better tribological property than $1 \#$ despite the similar dimple density, probably due to the smaller dimple spacing in the sliding direction of 2\#. The best anti-wear performance appeared on the condition of GNS-3\#, and the most probable cause is that micro-dimples can store the GNS additives.

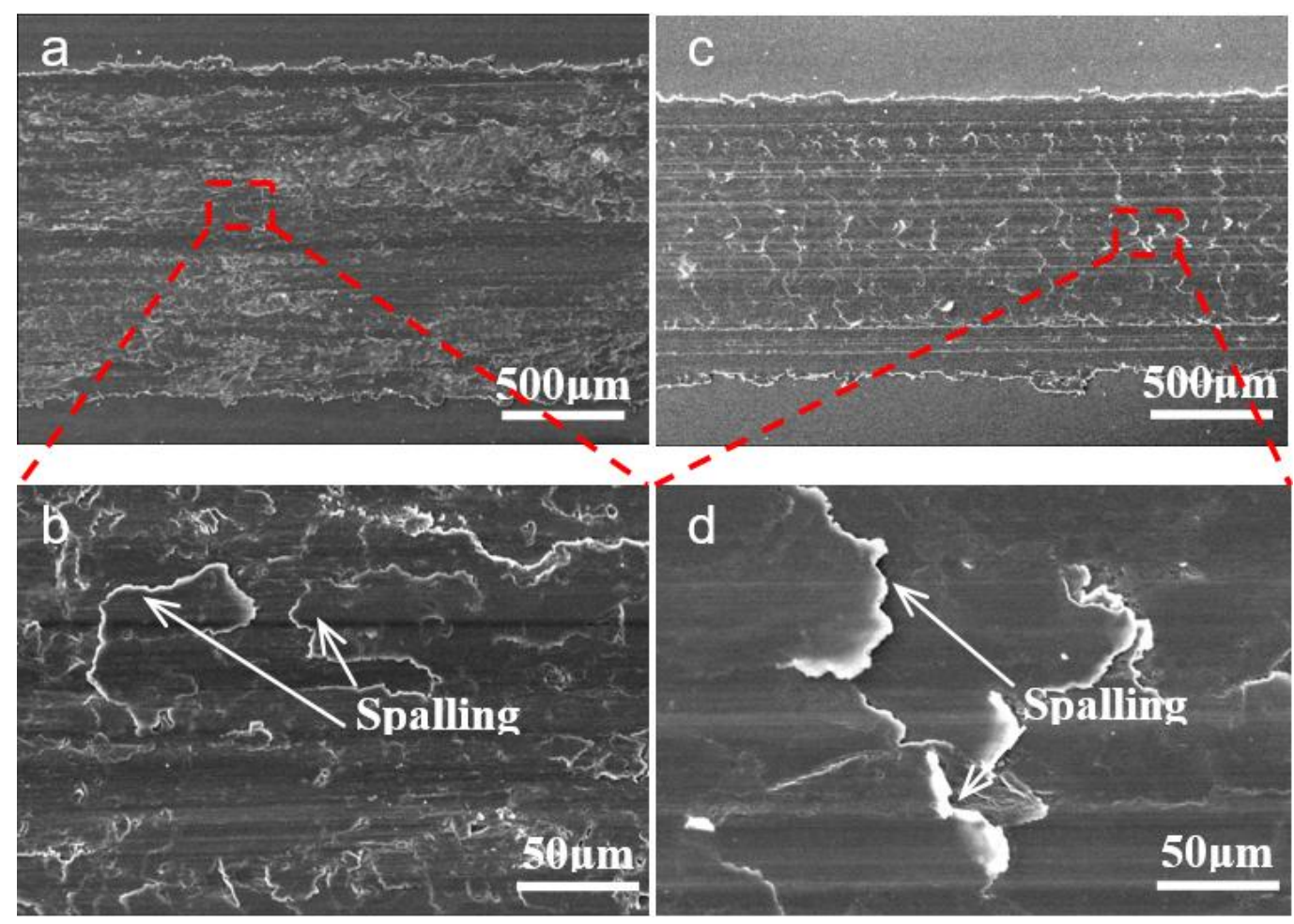

Fig. 7 SEM morphologies recorded at different magnifications of worn plate surfaces of $0 \#$ tested in

(a-b) PAO4 base oil and (c-d) GNS dispersed oil. 


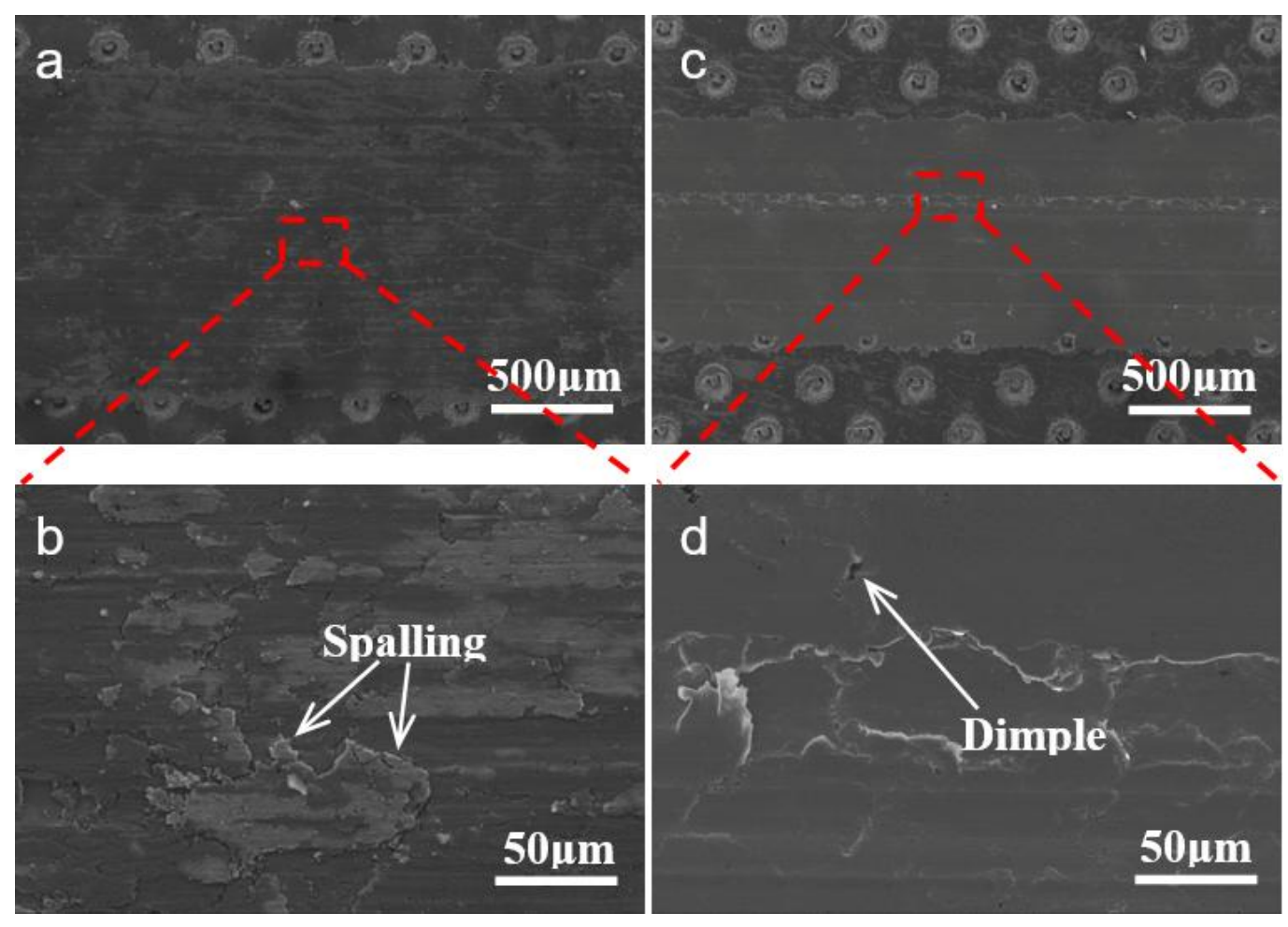

Fig. 8 SEM morphologies recorded at different magnifications of worn plate surfaces of $1 \#$ tested in (a-b) PAO4 base oil and (c-d) GNS dispersed oil.
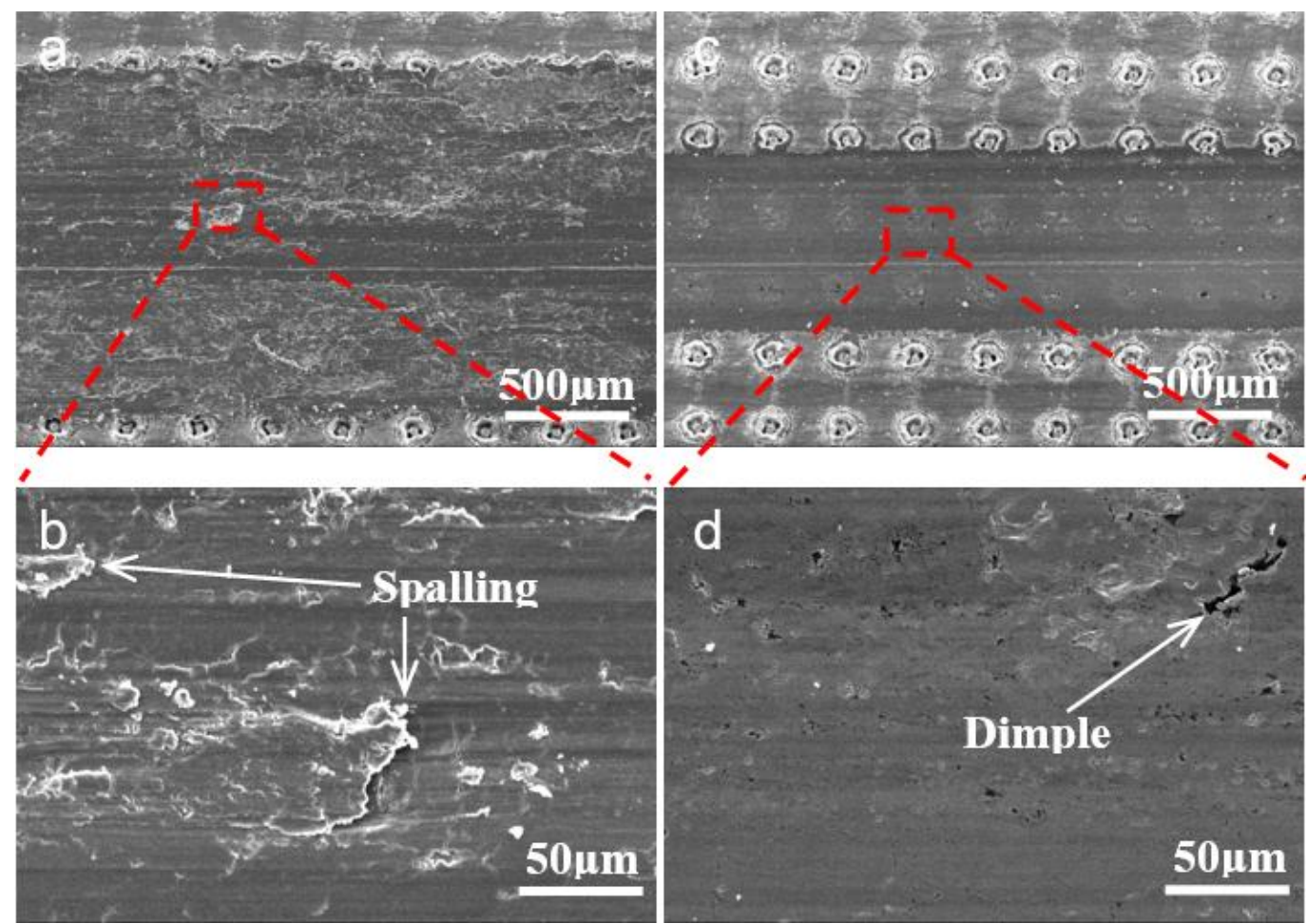

Fig. 9 SEM morphologies recorded at different magnifications of worn plate surfaces of 2\# tested in (a-b) PAO4 base oil and (c-d) GNS dispersed oil. 


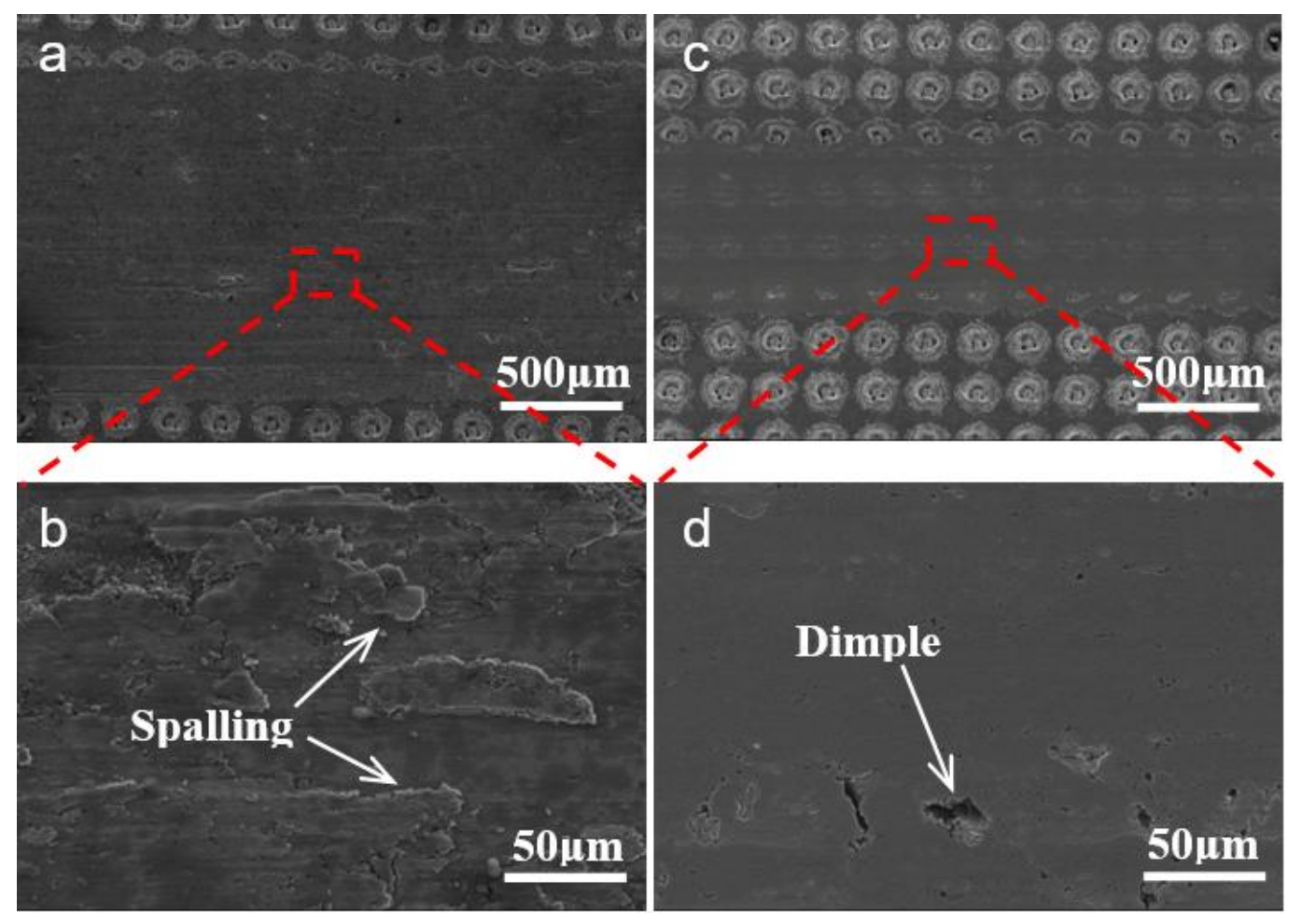

Fig. 10 SEM morphologies recorded at different magnifications of worn plate surfaces of $3 \#$ tested in (a-b) PAO4 base oil and (c-d) GNS dispersed oil.

\subsection{EDX and Raman analysis of friction contact regions}

To expound the wear reduction mechanisms in depth, local structures were characterized using energy-dispersive x-ray spectroscopy (EDX) and Raman spectroscopy. For 3\#, the EDX spectra obtained from the worn and un-contact area under GNS dispersed oil lubrication are shown in Fig. 11. Table 2 shows the elemental content corresponding to the EDX spectra in Fig. 11. It is obvious that $\mathrm{O}$ element appears on the worn area, while only $\mathrm{C}$ and $\mathrm{Fe}$ exist on the un-contact area. And the $\mathrm{C}$ content of the worn area is significantly higher than that of the un-contact area. In addition, it the highest when the analysis location was position 1 of the worn dimple (it increased to $53.17 \%$ from $31.49 \%$ compared with that in position 2 ). Thus, it inferred that the tribological properties of GNS may be ascribed to a protective film caused by adsorption of GNS to the surfaces of the sliding parts. Another evidence was provided by Raman measurements as fellow. 


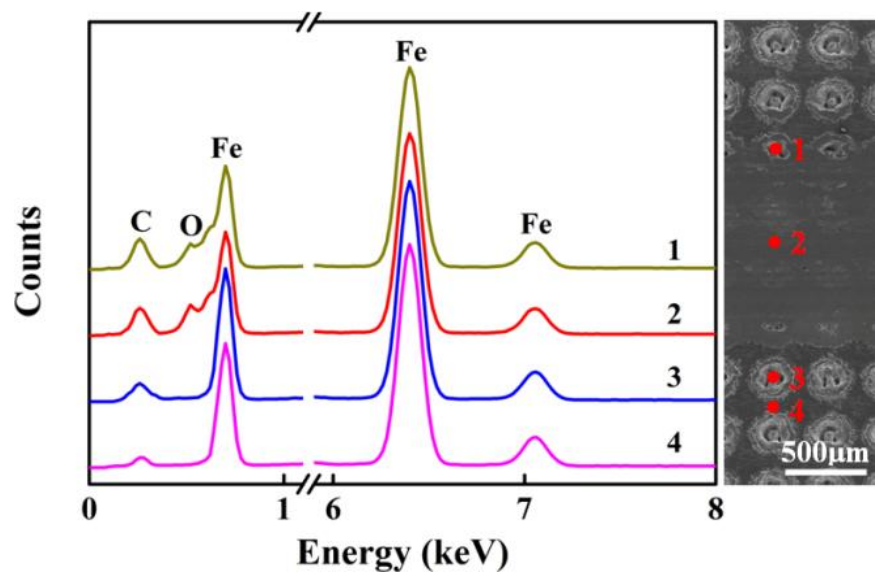

Fig. 11 EDX spectra obtained from different locations on the worn plate of $3 \#$. The right image indicates the approximate positions where EDX spectra were obtained.

Table 2 Elemental content corresponding to the EDX spectra in Fig. 11

\begin{tabular}{cccc}
\hline Positions & $\mathrm{C}(\%)$ & $\mathrm{O}(\%)$ & $\mathrm{Fe}(\%)$ \\
\hline 1 & 53.17 & 13.32 & 33.51 \\
2 & 31.49 & 16.20 & 52.31 \\
3 & 20.16 & 0 & 79.84 \\
4 & 9.76 & 0 & 90.24 \\
\hline
\end{tabular}

Fig. 12 shows the Raman spectra of different plate samples inside the wear scars and avoid the residual dimples under GNS dispersed oil lubrication. For all case, disordered GNS adsorbed on the sliding interfaces during the tribotests, as evidenced from the disappearance of 2D peak in the wear scars, which is consistent with the findings of EDX measurements. At the same time, the D and G peaks were observed, representing the typical characteristic of GNS additives. To compare the Raman spectra of different samples, Gaussian fitting was applied for the D and G peaks fitting (Fig. 13(a-d)). The fitting results of these two peaks as well as the intensity ratio of the D peak to $G$ peak $\left(I_{D} / I_{G}\right)$ are also shown in Fig. 13. As shown in Fig. 13(f-g), not only the intensity of the $D$ and $G$ peaks but also the $I_{D} / I_{G}$ follows the order: $0 \#<1 \#<2 \#<3 \#$. In the Raman spectra of graphene, the $I_{D} / I_{G}$ can be used to characterize the sp3/sp2 bonding ratio [31]. As indicated by Ferrari and Robertson's three-stage model for the Raman spectra of disordered and amorphous carbon [32], GNS additives on the textured surfaces underwent more significant graphitization than that on the original one during 
friction, probably attributed to the large amount of graphitized wear debris entrapped in the micro-dimples. The condition of GNS-3\# exhibited the most obvious graphitizing transformation after the friction testing, considering its largest dimple density. However, the $\mathrm{I}_{\mathrm{D}} / \mathrm{I}_{\mathrm{G}}$ of GNS-1\# condition was notably lower than that of GNS-2\# condition despite the similar dimple density, which is unexpected, but consistent with the results of the wear rates, probably due to the larger dimple spacing in the sliding direction of $1 \#$.

These results indicated that GNS displays excellent properties on reducing friction and wear as additives in PAO4 oil, especially evident on the textured surfaces. The low friction and wear reduction may be due to the 2D structure and extreme strength of graphene. Fig. 14 illustrates the effects of surface texturing and GNS additives on the friction and wear behaviour. In this study, a slow sliding speed of $5 \mathrm{~mm} / \mathrm{s}$ and a relatively high temperature of $100^{\circ} \mathrm{C}$ were employed and hence correspond to a boundary lubrication situation. The PAO4 base oil can be easily cut off due to the high contact stress achieved from the small contact zone under the ball-on-plate contact model on the untextured plate (Fig. 14a). Additionally, comparing with the original plate, the textured plates exhibit relatively high friction and wear increase in PAO4 base oil. A possible explanation is that the boundary lubricating film rupture caused by the local high stress on the dimple edge and, hence, provides high friction (Fig. 14b). After GNS adding, the graphene layers sheared at the sliding contact interfaces attributed to its 2D structure [33, 34], and the oil film strength was improved (Fig. 14c). On the textured surfaces, the micro-dimples can store the GNS additives and release and, hence, improves the contact state of the friction interfaces (Fig. 14d). The GNS additives adsorbed on the sliding interfaces, and a protective film may be formed as confirmed by the EDX and Raman measurements (Fig. 11-13). Furthermore, the micro-dimples resulted in high contact stress on the sliding interfaces. However, it is also responsible for graphitization of GNS additives and, hence, promotes the adsorption of GNS. It is a possible explanation for the significant combined effect of GNS additives and surface texturing on reducing friction and wear, which is closely related with GNS structure and texture patterns. 


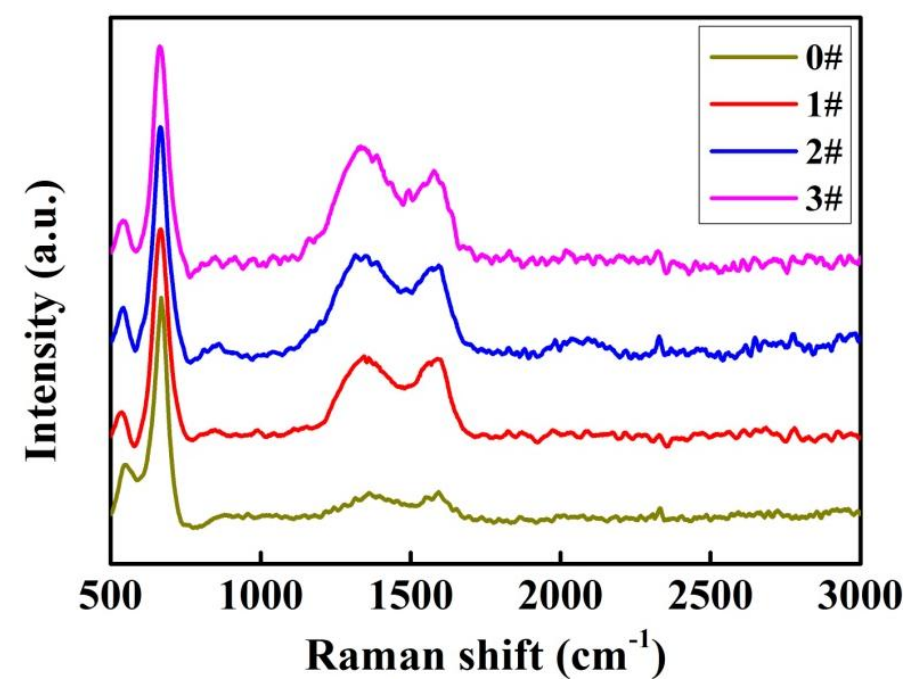

Fig. 12 Raman spectra of different plate samples inside the wear scar and avoid the residual dimple under GNS dispersed oil lubrication.

\section{Conclusions}

The combined effect of GNS as lubricating oil additives and laser surface texturing on reducing the friction and wear was investigated. High friction and wear increase was achieved under laser surface texturing alone affected because the high contact stress caused by the micro-dimples. At the same time, micro-dimples can store lubricating oil and trap wear debris, hence, reduce friction and wear. The GNS additives modified by Span-80 were well dispersed in PAO4 oil, and showed a good tribological properties (wear rate was reduced approximately $50 \%$ after adding GNS), especially on the textured surfaces (the reduction in wear was high at over 90\%). Condition of GNS-3\# showed the optimal tribological properties. These findings can be explained by the combined effect between micro-dimples and GNS additives: induce graphitizing transformation and the storing of GNS additives of the micro-dimples. It was confirmed by the characterization performed after tribotests, and indicating that the graphene layers sheared at the sliding contact interfaces, and a protective film was formed, which exhibited higher strength on the textured surfaces due to GNS storing and accelerating effect on the graphitization of GNS additives of micro-dimples. Furthermore, GNS structure and texture patterns are important factors influencing friction and wear properties. 


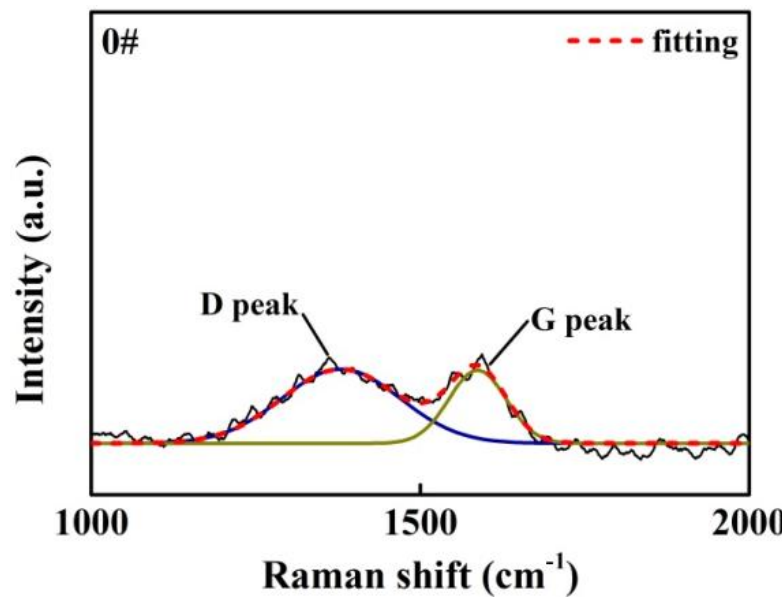

(a)

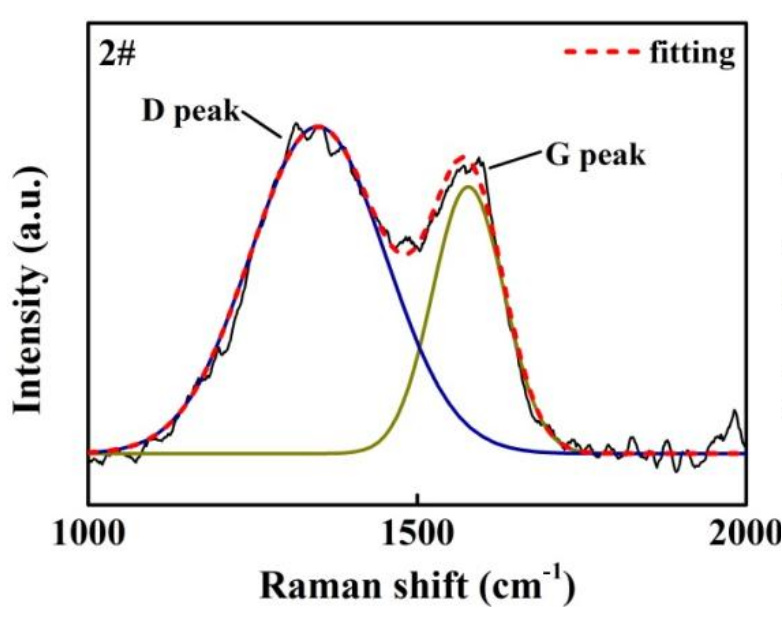

(c)

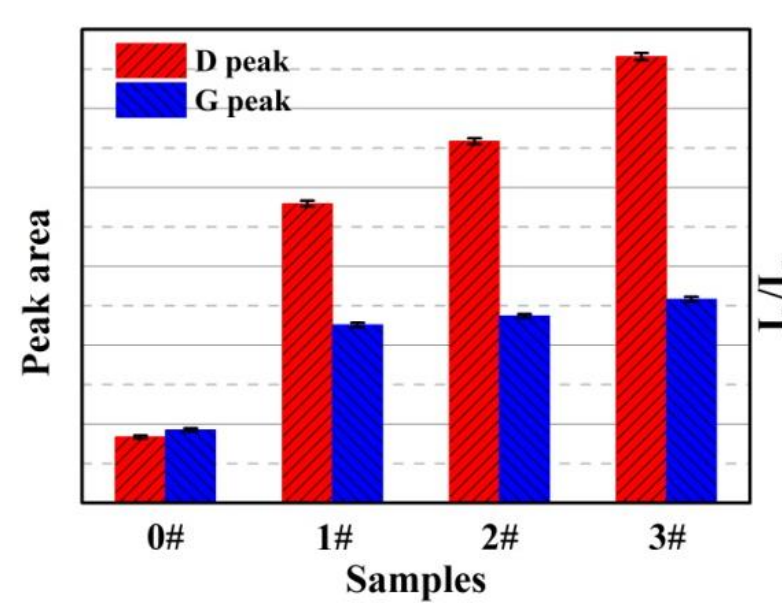

(e)

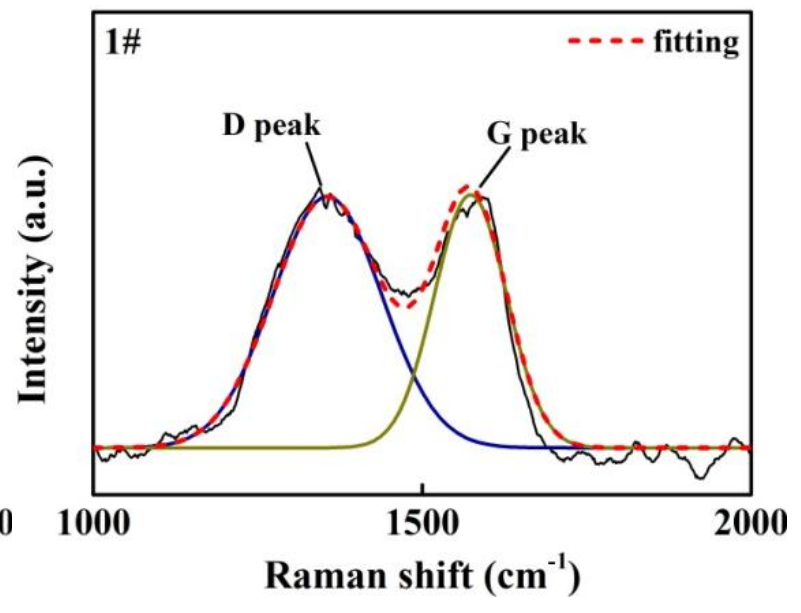

(b)

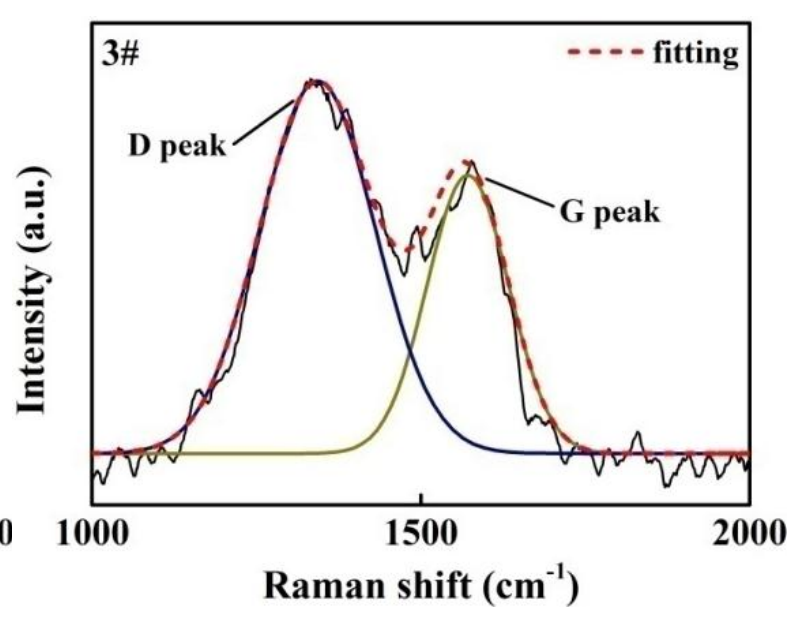

(d)

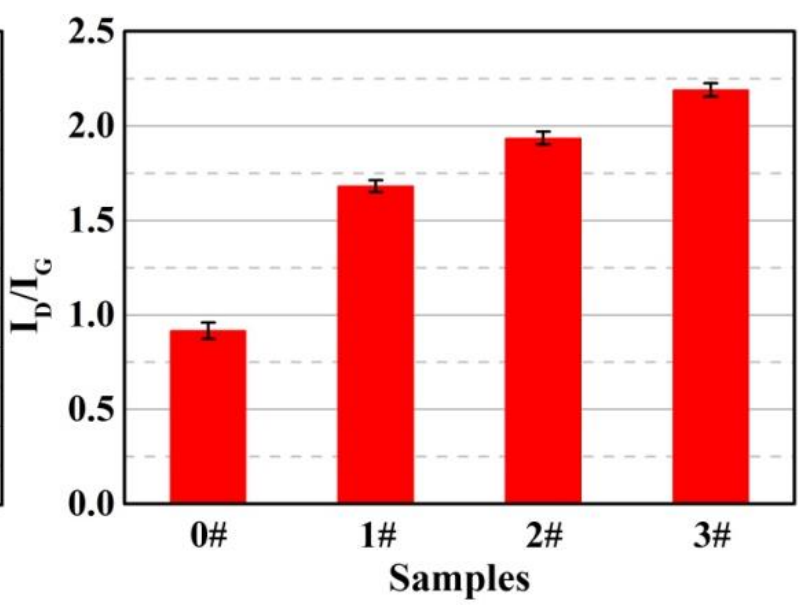

(f)

Fig. 13 Raman analysis. (a-d) Gaussian fitting applied for D and G peaks fitting of 0\#, 1\#, 2\# and 3\# respectively. (e-f) The fitting results of the D and G peaks as well as the intensity ratio of the D peak to $\mathrm{G}$ peak $\left(\mathrm{I}_{\mathrm{D}} / \mathrm{I}_{\mathrm{G}}\right)$. 


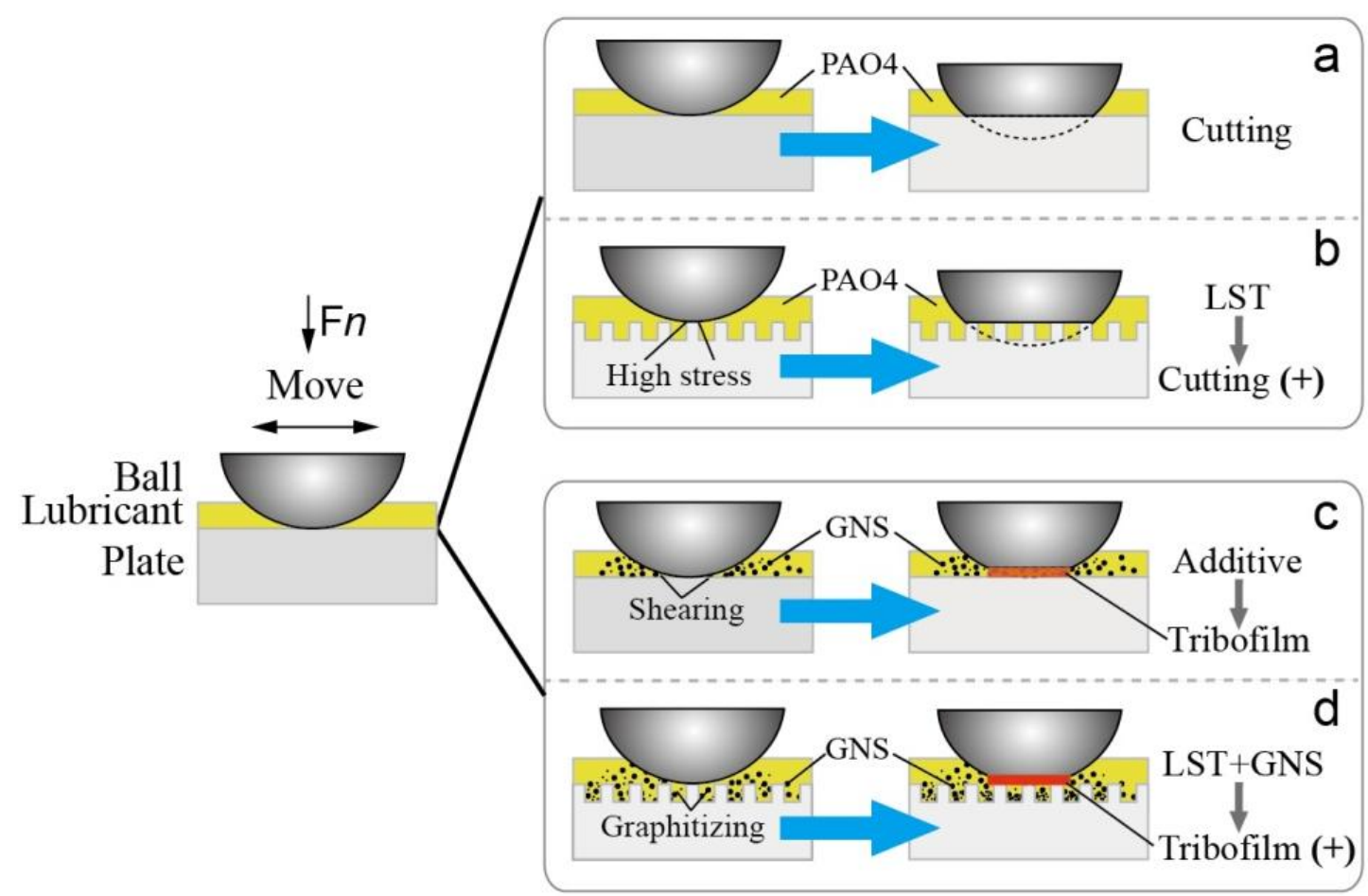

Fig. 14 Schematic diagram of the wear mechanism.

\section{Acknowledgements}

This study was supported by National Science Foundation of China (51375407 and U1530136). The authors thank Dr Wen Yue from China University of Geosciences in Beijing for providing the PAO4 oil. And thank Dr Jin-fang Peng from Southwest Jiaotong University for EDX measurements.

\section{References}

[1] Robert D. Heverly Trends in Tribology and Lubrication Engineering : 2014 Report on Emerging Issues and Trends[J]. Lubrication engineering, 2015, 10(1), 1-8. (in Chinese).

[2] Minami I. Ionic liquids in tribology[J]. Molecules, 2009, 14(6), 2286-2305.

[3] Berman D, Erdemir A and Sumant A V. Graphene: a new emerging lubricant[J]. Materials Today, 2014, 17(1), 31-42.

[4] Alazemi A A, Etacheri V, Dysart A D, et al. Ultrasmooth Submicrometer Carbon Spheres as Lubricant Additives for Friction and Wear Reduction[J]. ACS applied materials \& interfaces, 2015, 7(9), 5514-5521. 
[5] Yu B, Bansal D G, Qu J, et al. Oil-miscible and non-corrosive phosphonium-based ionic liquids as candidate lubricant additives[J]. Wear, 2012, 289, 58-64.

[6] Yu B, Liu Z, Ma C, et al. Ionic liquid modified multi-walled carbon nanotubes as lubricant additive[J]. Tribology International, 2015, 81, 38-42.

[7] Novoselov K S, Geim A K, Morozov S V, et al. Electric field effect in atomically thin carbon films[J]. science, 2004, 306(5696), 666-669.

[8] Zhang W, Zhou M, Zhu H, et al. Tribological properties of oleic acid-modified graphene as lubricant oil additives[J]. Journal of Physics D: Applied Physics, 2011, 44(20), 4329-4334.

[9] Kinoshita H, Nishina Y, Alias A A, et al. Tribological properties of monolayer graphene oxide sheets as water-based lubricant additives[J]. Carbon, 2014, 66, 720-723.

[10] Mao F, Wiklund U, Andersson A M, et al. Graphene as a lubricant on Ag for electrical contact applications[J]. Journal of Materials Science, 2015, 50(19), 6518-6525.

[11] Yitian P, Zhuoqiong W and Kun Z. Friction and Wear Properties of Different Types of Graphene Nanosheets as Effective Solid Lubricants[J]. Langmuir the Acs Journal of Surfaces \& Colloids, 2015, 31(28), 7782-91.

[12] Huang X, Qi X, Boey F, et al. Graphene-based composites[J]. Chemical Society Reviews, 2012, 41(2), 666-686.

[13] Xiao-Min Liu. Summary of texture research[J]. Application Research of Computers, $2008,8,011$.

[14] Zhang K, Deng J, Xing Y, et al. Effect of microscale texture on cutting performance of WC/Co-based TiAlN coated tools under different lubrication conditions[J]. Applied Surface Science, 2015, 326:107-118.

[15] Kovalchenko A, Ajayi O, Erdemir A, et al. The effect of laser texturing of steel surfaces and speed-load parameters on the transition of lubrication regime from boundary to hydrodynamic[J]. Tribology Transactions, 2004, 47(2), 299-307.

[16] Lorenzo-Martin C, Ajayi O O. Effect of SiC particle impact nano-texturing on tribological performance of 304L stainless steel[J]. Applied Surface Science, 2014, 315(315):287-291. 
[17] Malekian M, Mostofa M G, Park S S, et al. Modeling of minimum uncut chip thickness in micro machining of aluminum[J]. Journal of Materials Processing Technology, 2012, 212(3), 553-559.

[18] Byun J W, Shin H S, Kwon M H, et al. Surface texturing by micro ECM for friction reduction[J]. International Journal of Precision Engineering and Manufacturing, 2010, 11(5), 747-753.

[19] Etsion I. State of the art in laser surface texturing[J]. Journal of Tribology, 2005, 127(1), 248-253.

[20] Etsion I, Sher E. Improving fuel efficiency with laser surface textured piston rings[J]. Tribology International, 2009, 42(4), 542-547.

[21] Wan Y, Xiong D S. The effect of laser surface texturing on frictional performance of face seal[J]. Journal of materials processing technology, 2008, 197(1): 96-100.[22] Li D, Mueller M B, Gilje S, et al. Processable aqueous dispersions of graphene nanosheets[J]. Nature nanotechnology, 2008, 3(2), 101-105.

[23] Zhang W, He W, Jing X. Preparation of a stable graphene dispersion with high concentration by ultrasound[J]. The Journal of Physical Chemistry B, 2010, 114(32), 10368-10373.

[24] Kim H, Abdala A A, Macosko C W. Graphene/polymer nanocomposites[J]. Macromolecules, 2010, 43(16), 6515-6530.

[25] Ferrari A C, Meyer J C, Scardaci V, et al. Raman spectrum of graphene and graphene layers[J]. Physical review letters, 2006, 97(18), 13831-13840.

[26] Malard L M, Pimenta M A, Dresselhaus G, et al. Raman spectroscopy in graphene[J]. Physics Reports, 2009, 473(5), 51-87.

[27] Wang Y, Ni Z, Shen Z, et al. Interference enhancement of Raman signal of graphene[J]. Applied Physics Letters, 2008, 92(4), 043121-043121-3.

[28] Galda L, Sep J, Prucnal S. The effect of dimples geometry in the sliding surface on the tribological properties under starved lubrication conditions[J]. Tribology International, 2016, $99,77-84$.

[29] Dumitru G, Romano V, Weber H P, et al. Laser treatment of tribological DLC films[J]. Diamond and related materials, 2003, 12(3), 1034-1040. 
[30] Meng-fei Guo, Zhen-bing Cai, et al. Characterization and lubrication performance of diesel soot nanoparticles as oil lubricant additives[J]. RSC Advances, 2015, 5, $101965-101974$.

[31] Casiraghi C, Ferrari A C, Robertson J. Raman spectroscopy of hydrogenated amorphous carbons[J]. Physical Review B Condensed Matter, 2005, 72(8), 085401.

[32] Ferrari A C, Robertson J. Interpretation of Raman spectra of disordered and amorphous carbon[J]. Physical Review B Condensed Matter, 2000, 61(20), 14095-14107.

[33] Lin J, Wang L, Chen G. Modification of graphene platelets and their tribological properties as a lubricant additive[J]. Tribology Letters, 2011, 41(1), 209-215.

[34] Berman D, Erdemir A, Sumant A V. Reduced wear and friction enabled by graphene layers on sliding steel surfaces in dry nitrogen[J]. Carbon, 2013, 59, 167-175. 

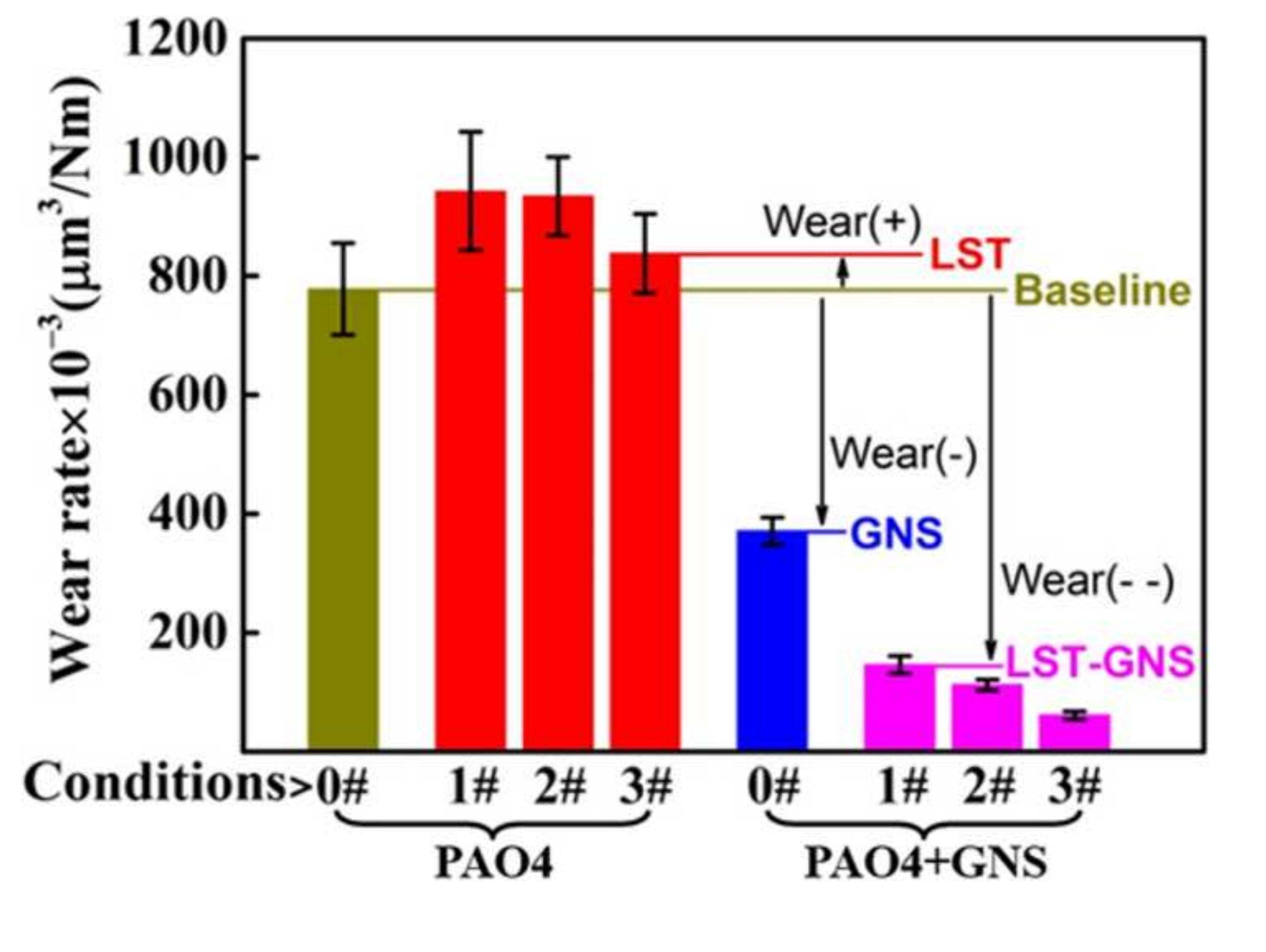